\title{
Responsive neurostimulation for the treatment of medically refractory epilepsy in pediatric patients: strategies, outcomes, and technical considerations
}

\author{
Armin Mortazavi, BS, ${ }^{1}$ Ross-Jordon S. Elliott, MD, ${ }^{2}$ Tiffany N. Phan, BA, ${ }^{3}$ John Schreiber, MD, ${ }^{3}$ \\ William D. Gaillard, MD, ${ }^{3}$ and Chima O. Oluigbo, MD $^{3}$
}

\begin{abstract}
${ }^{1}$ Georgetown University School of Medicine; ${ }^{2}$ Department of Neurological Surgery, George Washington University; and ${ }^{3}$ Department of Neurosurgery, Children's National Hospital, Washington, DC
\end{abstract}

\begin{abstract}
OBJECTIVE Children with medically refractory partial-onset epilepsy arising from eloquent cortex present a therapeutic challenge, as many are not suitable for resective surgery. For these patients, responsive neurostimulation may prove to be a potential tool. Although responsive neurostimulation has demonstrated utility in adults, little has been discussed regarding its utility in the pediatric population. In this study, the authors present their institution's experience with responsive neurostimulation via the RNS System through a case series of 5 pediatric patients.

METHODS A single-center retrospective study of patients who underwent RNS System implantation at Children's National Hospital was performed.

RESULTS Five patients underwent RNS System implantation. The mean patient age at treatment was 16.8 years, and the average follow-up was 11.2 months. All patients were considered responders, with a seizure frequency reduction of $64.2 \%$ without adverse events.

CONCLUSIONS All 5 patients experienced medium-term improvements in seizure control after RNS System implantation with decreases in seizure frequency $>50 \%$ from baseline preoperative seizure frequency. The authors demonstrated two primary configurations of electrode placement: hippocampal or amygdala placement via an occipitotemporal trajectory, as well as infratemporal surface electrodes and surface electrodes on the primary motor cortex. No adverse events were experienced in this case series.
\end{abstract}

https://thejns.org/doi/abs/10.3171/2020.11.PEDS20660

KEYWORDS responsive neurostimulation; medically refractory epilepsy; pediatric epilepsy

$\mathrm{F}$ EW effective therapeutic options are available for children living with medically refractory partialonset epilepsy. The likelihood of becoming seizure free with medication regimens alone is uncommon if two appropriately chosen antiseizure medications fail. ${ }^{1}$ This failure presents a major therapeutic challenge, as it is linked to significant comorbidities, such as cognitive impairment, depression, anxiety, and impaired activities of daily living. Although surgical interventions may be appropriate for a subset of these patients, nevertheless, many are not candidates for resective surgery if the seizure focus arises in eloquent cortex and cannot be safely removed. To this end, responsive neurostimulation has recently emerged as a safe and effective treatment for adult patients, although not much is known about this treatment modality in the pediatric setting. The RNS System (Neu-
roPace, Inc.) is an FDA-approved adjunctive treatment for adults aged 18 years and older with medically intractable partial-onset seizures arising from one or two foci. ${ }^{2-4}$

The RNS System is a closed-loop system designed to abort seizure activity. An electrical field is delivered to the brain when conditions are such that the device detects a potential seizure; this action relies on a predefined seizuredetection algorithm..$^{1-3,5-9}$ Initially, the RNS System was designed based on observations during functional mapping such that cortical electrical stimulation aborted epileptic cortical discharges. However, there is evidence that the RNS System may also act by other mechanisms, including altering plasticity of neural networks, suppressing cortical synchronization, and preventing epileptic activity. $2,5,6,10,11$

Positive evidence in seizure reduction has been demonstrated in clinical trials for adults, including a study that

ABBREVIATIONS EEG = electroencephalography; FCD = focal cortical dysplasia; fMRI = functional MRI; IEEG = intracranial EEG; MRE = medically refractory epilepsy; MST = multiple subpial transection; MTS = mesial temporal sclerosis; SEEG = stereo-EEG; VEEG = video-EEG; VNS = vagus nerve stimulation.

SUBMITTED August 2, 2020. ACCEPTED November 20, 2020.

INCLUDE WHEN CITING Published online April 30, 2021; DOI: 10.3171/2020.11.PEDS20660. 

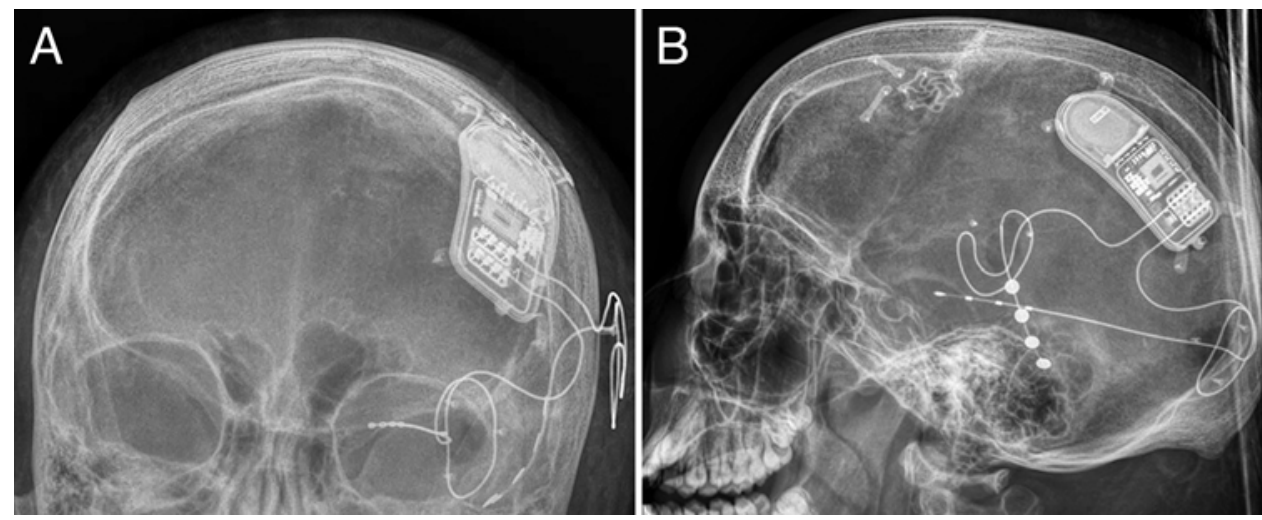

FIG. 1. Patient 1. Left: Anteroposterior skull radiograph of the RNS System showing a depth electrode in the left hippocampus implanted via an occipitotemporal trajectory, a left infratemporal subdural cortical strip electrode, and a pulse generator placed in the left parietal region. Right: Lateral skull radiograph view of the RNS System.

demonstrated a $43 \%$ responder rate (patients with $50 \%$ or greater reduction in seizures) at 1 year postimplantation..$^{2,4}$, $5,12,13$ However, the emergence of the RNS System as a therapeutic option for children with medically intractable partial-onset seizure remains elusive. ${ }^{8,14}$ In this study, we present our experience with the RNS System in 5 pediatric patients with medically intractable partial-onset epilepsy with particular focus on technical aspects and outcomes.

\section{Methods}

\section{Study Description and Data Collection}

After Children's National Hospital IRB approval, a single-center retrospective study reviewed pediatric patients who underwent RNS System implantation $(n=5)$. Each case was discussed at a multidisciplinary meeting, and the RNS System was considered a treatment option only after concluding that the patient had significant medically refractory epilepsy (MRE) involving eloquent cortex.

The following variables were collected: age, sex, handedness, age at epilepsy onset, preoperative seizure frequency, imaging findings, functional MRI (fMRI) findings, Wada test results, seizure onset zone, previous surgery, stereo-electroencephalography (SEEG) results, localization, target of implantation, surgical technique, complications, follow-up, postoperative seizure frequency, and reduction in seizure frequency. Seizure frequency reduction was calculated for all patients based on reported preoperative seizure frequency and postoperative seizure frequency. The study followed the Preferred Reporting of Case Series in Surgery guidelines to ensure appropriate reporting of the outcomes.

\section{Case Series}

\section{Patient 1}

A 16-year-old, right-handed female patient, who had not responded to a vagus nerve stimulation (VNS), presented with a 5-year history of MRE. The patient's seizure onset began at 11 years of age, and she reportedly experienced approximately 7 seizures per week. Typical seizures included focal unaware seizures with variable semiology, including eye rolling, head turning to the right, right-sided automatisms, and occasional secondarily generalized tonic-clonic seizures. To further localize her seizures, she underwent robot-assisted bilateral stereotactic implantation of depth electrodes for invasive monitoring. During 7 days of monitoring, the patient experienced multiple stereotypical seizures. The SEEG recordings were notable for ictal and interictal activity primarily in the superficial electrodes in the left hippocampus and left amygdala. Wada testing with methohexital injections revealed the right hemisphere was unable to support memory function, thus indicating that the left hemisphere/hippocampus was critical for verbal memory. Furthermore, language mapping established left hemispheric dominance for language function.

Although her left hippocampus was determined to be the epileptic focus, resection or laser ablation was deemed inappropriate as it was critical for her verbal memory. Following extensive conversation, a consensus between the family and the epilepsy team was reached to undergo off-label use of the RNS System in a bid to control her medically refractory seizures, which were significantly impacting her quality of life. At surgery, using the Leksell stereotactic head frame, stereotactic implantation of a NeuroPace depth electrode was achieved in the left hippocampus and secondary left infratemporal lobe subdural strip electrode via an occipitotemporal trajectory and temporal burr hole, respectively. The NeuroPace pulse generator was placed in the left parietal region (Fig. 1). The patient had an uncomplicated postoperative course during her 19-month follow-up. Postoperatively, this patient experienced 14 seizures per month, reduced from 7 seizures per week, indicating a 50\% reduction in seizures.

\section{Patient 2}

A 17-year-old, right-handed male patient with a history of attention deficit disorder presented with a 10 -year history of focal MRE. He experienced cluster seizures roughly 3 days per week. Following review at the epilepsy multidisciplinary meeting, he first underwent ROSA (Zimmer Biomet) electroencephalography (EEG) depth electrode placement in the left frontal and temporal brain lobes for 

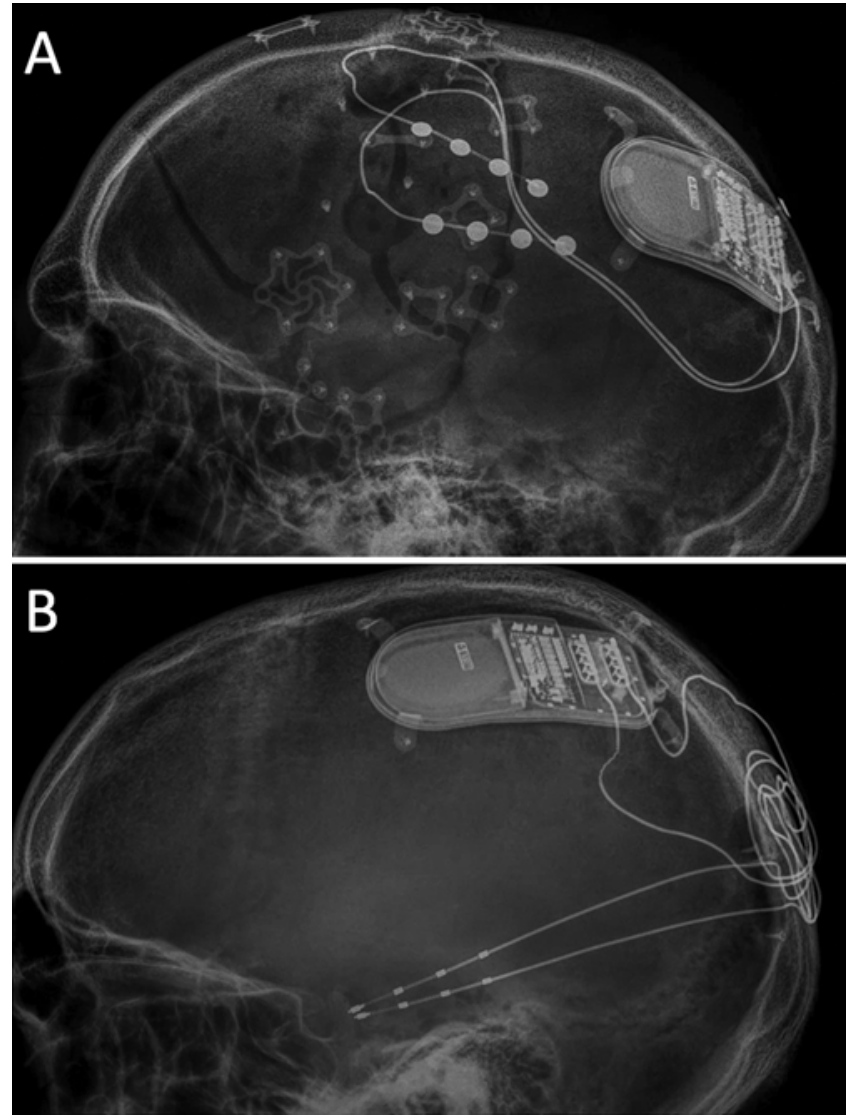

FIG. 2. A: Patient 2. Lateral skull radiograph of the RNS System with subdural cortical strip electrodes placed over the epileptic focus in the left primary motor cortex and a pulse generator placed in the left parietal region. B: Patient 3. Lateral skull radiograph of the RNS System showing depth electrodes implanted in the left hippocampus and right hippocampus via an occipitotemporal trajectory and a pulse generator in the left parietal region.

localization of seizure focus. This investigation suggested that his seizures were arising around the left middle frontal gyrus. He subsequently underwent a left craniotomy for grid electrode implantation on the left frontal convexity and inferior frontal subdural strip to define the suspected epileptic focus.

Additionally, extraoperative language mapping and fMRI revealed left hemispheric dominance for language. Over a 7-day period of invasive intracranial monitoring, the seizures were discovered to come from contact F63 of the large frontotemporal grid. Subsequently, cortical brain stimulation and mapping showed that contact F63 was part of the left primary motor cortex and responsible for tongue movement. As his seizures were arising from the primary motor cortex, it was decided that he was not an appropriate candidate for resective epilepsy surgery. The decision was made to proceed with off-label placement of the RNS System.

Following a left parietal craniotomy and localization with Brainlab neuronavigation, RNS subdural cortical strip electrodes were placed over the epileptic focus in the left primary motor cortex, and the pulse generator was implanted in the left parietal region (Fig. 2A). Twentytwo months after implantation, the patient reported experiencing 2 to 3 seizures per month, decreased from 4 to 5 seizures approximately 3 days per week, with shorter duration and less severity, although seizure recovery time remained unchanged, signifying a $75 \%$ reduction in seizure frequency.

\section{Patient 3}

A 15-year-old male patient with an onset of focal MRE at 9 months of age presented with 4 to 5 cluster seizures per week. Brain MRI demonstrated marked left mesial temporal sclerosis (MTS) and loss of architecture in the right hippocampus with suspicion of right temporal pole focal cortical dysplasia (FCD). The patient underwent a 7-day SEEG study that revealed bilateral independent hippocampus/amygdala seizures, with seizures on the right side occurring far more frequently than on the left. Additionally, the seizures originating on the right frequently spread to the left hippocampus, resulting in his stereotypical seizures. His fMRI studies revealed that language predominantly originated in the left hemisphere.

The patient underwent implantation of bilateral hippocampal RNS depth electrodes in the left hippocampus and right hippocampus via an occipital temporal trajectory (Fig. 2B) using the Leksell stereotactic head frame. After 6 months of follow-up, the patient experienced seizurefree periods for 2 to 3 weeks and subsequent 2 to 3 cluster seizures with unchanged semiology, resulting in a $66 \%$ seizure frequency reduction.

\section{Patient 4}

A 16-year-old male patient with a history of autism and anxiety disorder presented with a lifelong history of focal unaware MRE secondary to left-sided MTS. The patient experienced his first seizure, involving motor activity on the right side, 6 hours after birth. The seizures were controlled with phenobarbitone by the patient's 3rd day of life to 4 years of age, when he was taken off this medication. He remained seizure free for another 4 years until his seizures recurred and persisted at the age of 8 years.

Brain MRI showed bilateral frontal encephalomalacia. Furthermore, FDG PET demonstrated a large area of decreased FDG uptake involving much of the left temporal lobe as well as the bifrontal regions in the area of the encephalomalacia. Following discussion, the patient ultimately underwent ROSA implantation of SEEG depth electrodes in the left frontal and temporal lobes. Seven-day invasive intracranial monitoring exhibited 3 stereotypical seizures electrographically arising in the left amygdala and left hippocampus electrodes.

The fMRI studies revealed left-side-dominant language function, and Wada testing suggested minimal capacity of the right hemisphere to support memory performance when the left hemisphere was compromised. Thus, surgical ablation or resection of the left hippocampus and amygdala was not considered a reasonable surgical option. The patient was deemed a candidate for off-label implantation of the RNS System.

At surgery, a NeuroPace depth electrode was placed in 

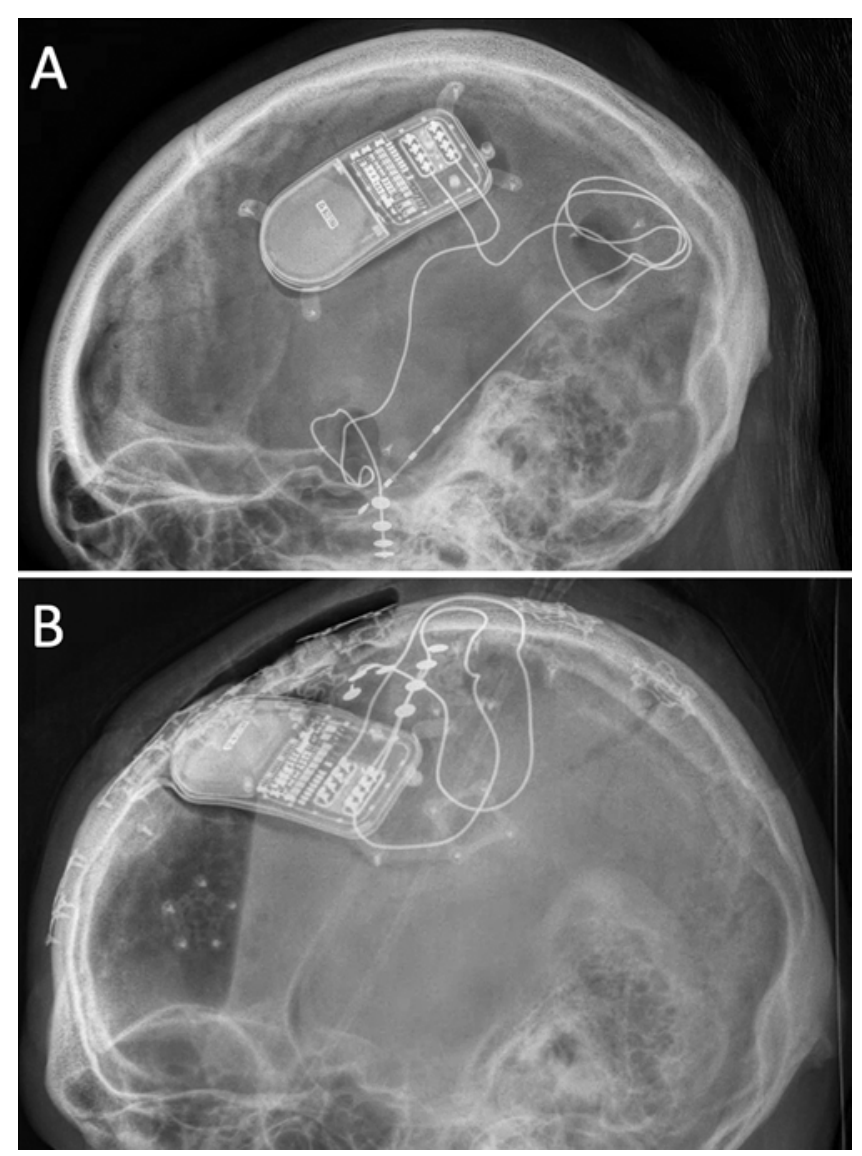

FIG. 3. A: Patient 4. Lateral skull radiograph of the RNS System showing a depth electrode placed in the left amygdala and hippocampus via an occipitotemporal approach and a secondary subdural cortical strip electrode placed over the left middle and inferior temporal gyri. B: Patient 5. Lateral skull radiograph of the RNS System with subdural cortical strip electrodes placed over the epileptic focus in the right primary motor cortex and a pulse generator placed in the right parietal region.

the left amygdala and hippocampus via an occipitotemporal approach, and a secondary subdural electrode was placed over the left middle and inferior temporal gyri (Fig. $3 \mathrm{~A})$. At 6 months of follow-up, the patient's seizures reduced to one every 2 weeks, indicating a $50 \%$ reduction in seizure frequency. Concerningly, although the focal seizures appeared to resolve, his generalized tonic-clonic seizures lasted for longer periods of time with a longer postictal period, albeit less frequently.

\section{Patient 5}

A 17-year-old male patient, who was diagnosed with MRE at 13 months of age, presented with a complicated neurological history. He had a history of Lennox-Gastaut syndrome and right FCD that required multiple epileptic resections of the right frontal lobe. Following two resective surgeries, his seizures increased in frequency and evolved to include drop seizures, resulting in further injuries. At presentation, he was experiencing up to 10 seizures per day with diverse semiology.

Video-EEG (VEEG) test results were consistent with Lennox-Gastaut syndrome, specifically slowing of the pos- terior basic rhythm and widespread slow activity with preponderance in the right temporal and left frontal regions correlating with disorganized sleep and the appearance of tonic seizures. Brain MRI showed evidence of previous right frontal lobe resections with residual dysplasia in the right inferior frontal gyrus posterior to the prior resection and extending to the right motor cortex. Acknowledging the impact on his quality of life, the patient underwent repeat right frontal craniotomy for safe resection of the residual area of cortical dysplasia while sparing right motor cortex involvement, anterior half corpus callosotomy, and off-label placement of the RNS System with two NeuroPace subdural strips over the motor cortex (Fig. 3B). The RNS System was used because of dysplasia involving the motor cortex, which could not be safely resected without significant morbidity.

At 3 months postsurgery, the patient's mother reported $80 \%$ improvement in his seizures with complete resolution of his drop seizures, which was likely related to the anterior corpus callosotomy. Nonetheless, it is difficult to determine how much of this improvement is directly from the RNS System or from resection.

\section{Results}

Five patients underwent RNS System implantation between June 2018 and February 2020. The overall mean age of the cohort was 16.8 years. The mean age at seizure onset was 2.7 years, and the mean follow-up was 11.2 months. Three of the 5 patients had seizure onset localization in the temporal lobe, and 2 patients had seizure onset localization in the primary motor cortex.

\section{Demographic, Clinic, and Preoperative Data}

Regarding our case series, all 5 patients met the criteria for implantation of the RNS System. Three patients had not undergone previous surgery prior to RNS System implantation; however, one patient previously underwent VNS implantation, and another patient previously underwent two right frontal lobe resections for cortical dysplasia. Localization of the seizure focus/foci was based on scalp EEG monitoring in all patients and intracranial EEG (IEEG) monitoring in 4 of 5 patients with normal MRI findings. Two patients had foci in the left amygdala and hippocampus; 1 patient had foci in the left primary motor cortex; 1 patient had foci in bilateral amygdala and hippocampi; and 1 patient had Lennox-Gastaut syndrome-like electrographic changes with foci in the right frontal opercula, right premotor cortex, and right motor primary cortex.

Functional testing included fMRI performed in 3 patients (one patient was unable to undergo these tests due to prior VNS implantation, and another patient was unable to cooperate for fMRI evaluation due to significant developmental delay) and Wada testing for 2 patients to predict the impact of hippocampal ablation on their verbal memory performance. Further details regarding patient characteristics are demonstrated in Table 1.

\section{Invasive Monitoring to Define Seizure Focus and RNS System Electrode Configuration}

Four of 5 patients underwent SEEG placement, and 1 


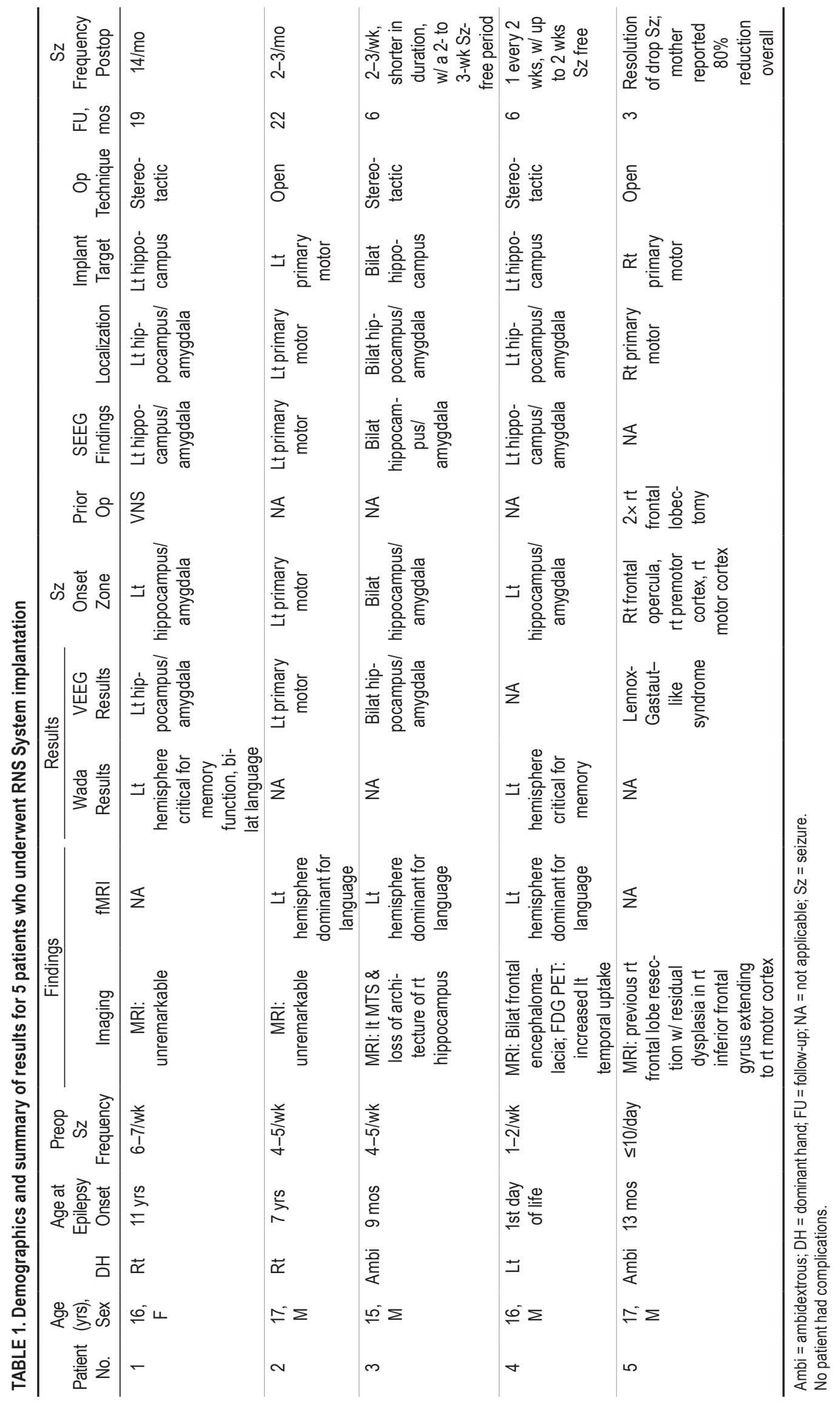




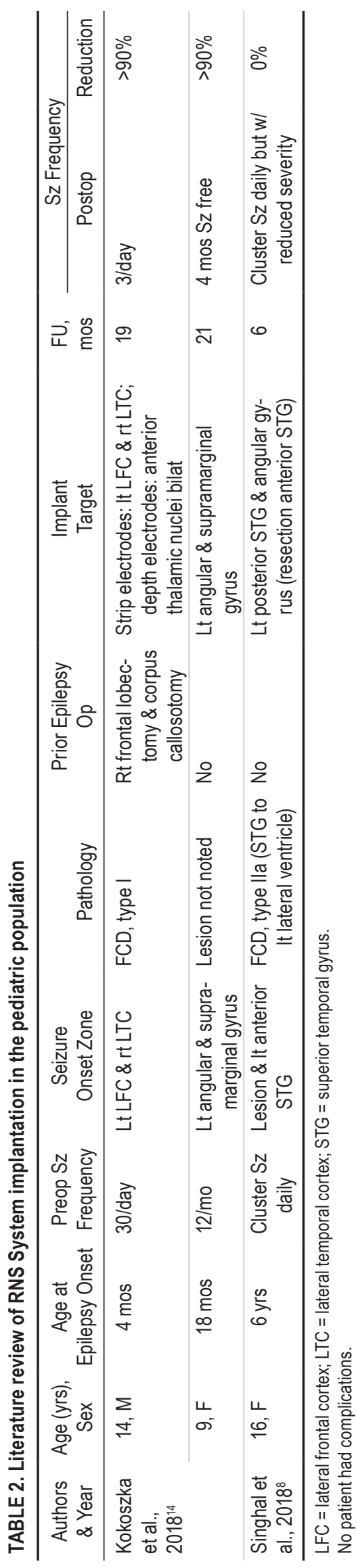

patient had SEEG followed by craniotomy for subdural grid placement and extraoperative brain mapping to define the relationship of his epileptic focus to his motor cortex (Table 1, patient 2). Multiple depth electrode placement strategies were employed based on preoperative data, and implantation strategy was specifically designed to interrogate a preoperative hypothesis of that patient's epileptic focus and seizure propagation paths.

NeuroPace electrode implantation configuration in these patients demonstrated two distinct subgroups: 1) a NeuroPace depth electrode was implanted in the hippocampus with or without a subdural strip electrode implanted over the temporal lobe; and 2) NeuroPace subdural strip electrodes were implanted over the primary motor cortex. Table 1 further describes the details regarding seizure focus and electrode configuration.

\section{Surgical Techniques for RNS System Implantation}

Two distinct strategies were employed for RNS System depth electrode implantation: stereotactic versus open implantation.

Three patients underwent stereotactic implantation utilizing a Leksell stereotactic head frame and implantation of depth electrodes with ROSA. One patient underwent an additional temporal burr hole for a subdural strip electrode. Furthermore, 2 patients underwent open implantation following craniotomy, with 1 involving a dysplastic cortex resection.

\section{Discussion}

MRE presents a complex clinical challenge for the pediatric population, especially for children with the seizure onset zone in eloquent cortex. We present our experience of pediatric patients with MRE partial-onset seizures who were unsuitable candidates for resective surgery but positively responded to RNS System device implantation. All 5 patients experienced medium-term improvements in seizure control after implantation, with decreases in seizure frequency of at least $50 \%$ from baseline preoperative seizure frequency. No intraoperative or postoperative complications were experienced.

Responsive neurostimulation has demonstrated efficacy in decreasing seizure frequency as well as improving cognitive outcomes in adults with MRE partial-onset seizures. ${ }^{5,6,12,15} \mathrm{~A}$ study with up to 6 years of follow-up demonstrated seizure frequency reduction ranging from $48 \%$ to $66 \%$ after RNS System implantation with a $64 \%$ responder rate. Our study and previous case reports ${ }^{1,14}$ demonstrated efficacy in seizure reduction with the RNS System in pediatric populations. To this end, it may be possible to extrapolate from adult studies to the pediatric population. Nonetheless, large-scale randomized clinical trials in the pediatric population would provide further clarification.

To our knowledge, there have been only 2 case reports discussing the use of responsive neurostimulation in the pediatric population (Table 2). Kokoszka et al. reported their experience with 2 patients. ${ }^{14}$ One case was of a 14-year-old male patient with MRE and a history of severe developmental delay with pathological evidence of a type 
I FCD following a right frontal lobectomy. This patient experienced atonic seizures that resolved with a corpus callosotomy, but subsequently the patient developed increasing developmental and behavioral decline as well as increased generalizing seizures that were not controlled with VNS. Following seizure onset zone localization to the left lateral frontal and right lateral temporal cortex, RNS System strip electrodes were utilized. Additionally, depth electrodes were placed in the anterior thalamic nuclei bilaterally for deep brain stimulation trials. Initially, responsive neurostimulation was applied only to the cortical strips, resulting in a reduction of seizure frequency and severity from 30 to 3 seizures per day at the 19-month follow-up. However, seizure response was not optimal and thus the patient underwent corticothalamic stimulation trials, which, interestingly, resulted in 50\% further seizure reduction after 1 month. The second case was that of a 9-year-old, right-handed female patient who had experienced MRE beginning when she was 18 months old. Mapping localized an epileptogenic zone in the left posterior frontal and parietal lobes, which were suppressible with higher-amplitude stimulations, designating it as an appropriate responsive neurostimulation target. The patient underwent placement of cortical strips in the angular and supramarginal gyrus regions. At 21 months postoperatively, the patient had been seizure free for 4 months.

Furthermore, Singhal et al. reported performing responsive neurostimulation in a 16-year-old, right-handed female patient with a 10 -year history of MRE. ${ }^{8}$ Brain MRI suggested transmantle FCD extending from the superior temporal gyrus to the left lateral ventricle. IEEG revealed the seizure onset zone from lesion depth electrodes as well as the anterior superior temporal gyrus. Cortical mapping following stimulation of the posterior superior temporal gyrus and angular gyrus resulted in language disruption, while the anterior superior temporal gyrus did not. For this reason, the authors elected to resect the seizure focus on the anterior superior temporal gyrus which revealed FCD, and the RNS System was utilized for the remaining seizure foci. The authors reported that at the 6-month follow-up, the patient continued to experience daily seizures but with less severity and without losing awareness.

Although additional studies are necessary, our study suggests that responsive neurostimulation should be considered for pediatric patients in an attempt to reduce morbidity due to MRE. Our case series demonstrated an average seizure frequency reduction of $64.2 \%$ with no adverse events over an average of 11.2 months of follow-up. Common adverse events in the adult literature after implantation of the RNS System were implant site infection (9\%) and neurostimulator explantation (4.7\%). ${ }^{16}$ Postimplantation outcomes in adults continued to improve over time. ${ }^{6}$ Although it has yet to be investigated in the pediatric population, increased ability for network plasticity in children may lead to added improvement. ${ }^{17}$ There is evidence that long-term stimulation may result in modulation of seizure network activity rather than solely the direct effects on each detected seizure. ${ }^{6,9,10}$ This further demonstrates that earlier intervention with responsive neurostimulation in children may be a sustainable treatment option.

In adults, long-term responsive neurostimulation is as- sociated with benefits in neuropsychological outcomes. With a 5-year follow-up in an adult cohort, Quality of Life In Epilepsy Inventory-89 assessments demonstrated significant improvements in attention, language, verbal learning, memory, social function, and overall quality of life..$^{12,16}$ Certainly, this is important in the pediatric population as the majority of antiepileptic drug medications are halted due to side effects, including those that are cognitive and behavioral, which perhaps are improved with responsive neurostimulation. ${ }^{18}$ Improvements in neuropsychological outcomes would unmistakably be valuable for pediatric patients with MRE.

In order to determine the seizure onset zone, our institution utilized MRI, VEEG, SEEG, and IEEG. These techniques coupled with functional testing-Wada tests, fMRI, and language and motor mapping-to determine the position with regard to functional areas, allowed us to determine safe, potentially therapeutic options. All 5 patients could not undergo full epileptic resection without significant risk. However, patient 5 was determined to benefit from partial resective surgery and a corpus callosotomy to address his drop seizures. The benefit of partial resection in combination with neurostimulation is yet to be defined, but longer follow-up may provide guidance.

Patients and their families were counseled on all potential treatments, which included furthering pharmacological management or multiple subpial transection (MST), as resection was not a safe treatment modality given their seizure focus arising from eloquent tissue. Indications, risks, alternatives, palliative measures, MRI restrictions, and periodic pulse generator changes were described in depth. Procedures were explained utilizing pictures and models. All questions were answered until the patients and/or their families verbalized that they had a good understanding of the procedure.

Prior to responsive neurostimulation, MST may have been employed to address concerns of proximity and involvement of eloquent cortex. ${ }^{19}$ Although MST has been shown to be beneficial, adults have demonstrated greater benefit from responsive neurostimulation when epileptic regions involve eloquent cortex..$^{20}$ In addition, responsive neurostimulation has an added advantage of its reversibility and adjustability, unlike MST, which is a lesional irreversible procedure.

Although responsive neurostimulation via the RNS System is a potentially safe and effective option for children with MRE, limitations exist and have been demonstrated in adult populations. While RNS System implantation has not proven to be curative in the adult population, it is palliative and effective in reducing seizure burden. Additionally, the RNS System requires implantation in fullthickness craniectomy, which may present a challenge in pediatric patients with smaller, growing skulls. Finally, the RNS System is not FDA approved for use in the pediatric population, which may affect patient payer insurance approval.

\section{Conclusions}

The RNS System may be a safe and effective treatment option for children with MRE. Our single-institution case 
series demonstrated seizure frequency reduction with no complications in all 5 patients. We reported our technical experience, which included two primary configurations of electrode placement: depth electrodes for hippocampus or amygdala placement via an occipitotemporal trajectory, and infratemporal surface electrodes and surface electrodes on the primary motor cortex. Our case series is limited, as it is retrospective and included only 5 patients. Further studies are warranted to expand understanding of the use of RNS System implantation in children. Longterm follow-up on these patients and quantitative electrocorticographic data regarding changes in recorded epileptic activity would provide insight and help define which patients are most likely to benefit from therapy.

\section{References}

1. Karsy M, Guan J, Ducis K, Bollo RJ. Emerging surgical therapies in the treatment of pediatric epilepsy. Transl Pediatr. 2016;5(2):67-78.

2. Skarpaas TL, Jarosiewicz B, Morrell MJ. Brain-responsive neurostimulation for epilepsy (RNS ${ }^{\circledast}$ System). Epilepsy Res. 2019;153:68-70.

3. Yan H, Toyota E, Anderson M, et al. A systematic review of deep brain stimulation for the treatment of drug-resistant epilepsy in childhood. J Neurosurg Pediatr. 2018;23(3):274-284.

4. Heck CN, King-Stephens D, Massey AD, et al. Two-year seizure reduction in adults with medically intractable partial onset epilepsy treated with responsive neurostimulation: final results of the RNS System Pivotal trial. Epilepsia. 2014;55(3): 432-441.

5. Geller EB, Skarpaas TL, Gross RE, et al. Brain-responsive neurostimulation in patients with medically intractable mesial temporal lobe epilepsy. Epilepsia. 2017;58(6):994-1004.

6. Kokkinos V, Sisterson ND, Wozny TA, Richardson RM. Association of closed-loop brain stimulation neurophysiological features with seizure control among patients with focal epilepsy. JAMA Neurol. 2019;76(7):800-808.

7. Roland JL, Smyth MD. Recent advances in the neurosurgical treatment of pediatric epilepsy: JNSPG 75th Anniversary Invited Review Article. J Neurosurg Pediatr. 2019;23(4): 411-421.

8. Singhal NS, Numis AL, Lee MB, et al. Responsive neurostimulation for treatment of pediatric drug-resistant epilepsy. Epilepsy Behav Case Rep. 2018;10:21-24.

9. Starnes K, Miller K, Wong-Kisiel L, Lundstrom BN. A review of neurostimulation for epilepsy in pediatrics. Brain Sci. 2019;9(10):E283.

10. Sohal VS, Sun FT. Responsive neurostimulation suppresses synchronized cortical rhythms in patients with epilepsy. Neurosurg Clin N Am. 2011;22(4):481-488, vi.

11. Lee B, Zubair MN, Marquez YD, et al. A single-center experience with the NeuroPace RNS System: a review of techniques and potential problems. World Neurosurg. 2015;84(3): 719-726.
12. Loring DW, Kapur R, Meador KJ, Morrell MJ. Differential neuropsychological outcomes following targeted responsive neurostimulation for partial-onset epilepsy. Epilepsia. 2015; 56(11):1836-1844.

13. Meador KJ, Kapur R, Loring DW, et al. Quality of life and mood in patients with medically intractable epilepsy treated with targeted responsive neurostimulation. Epilepsy Behav. 2015;45:242-247.

14. Kokoszka MA, Panov F, La Vega-Talbott M, et al. Treatment of medically refractory seizures with responsive neurostimulation: 2 pediatric cases. J Neurosurg Pediatr. 2018;21(4): 421-427.

15. Morrell MJ. Responsive cortical stimulation for the treatment of medically intractable partial epilepsy. Neurology. 2011; 77(13):1295-1304.

16. Bergey GK, Morrell MJ, Mizrahi EM, et al. Long-term treatment with responsive brain stimulation in adults with refractory partial seizures. Neurology. 2015;84(8):810-817.

17. Mundkur N. Neuroplasticity in children. Indian J Pediatr. 2005;72(10):855-857.

18. Bootsma HP, Ricker L, Hekster YA, et al. The impact of side effects on long-term retention in three new antiepileptic drugs. Seizure. 2009;18(5):327-331.

19. Benifla M, Otsubo H, Ochi A, et al. Multiple subpial transections in pediatric epilepsy: indications and outcomes. Childs Nerv Syst. 2006;22(8):992-998.

20. Jobst BC, Kapur R, Barkley GL, et al. Brain-responsive neurostimulation in patients with medically intractable seizures arising from eloquent and other neocortical areas. Epilepsia. 2017;58(6):1005-1014.

\section{Disclosures}

The authors report no conflict of interest concerning the materials or methods used in this study or the findings specified in this paper.

\section{Author Contributions}

Conception and design: Oluigbo. Acquisition of data: Oluigbo, Elliott, Phan. Analysis and interpretation of data: Oluigbo, Mortazavi, Elliott, Schreiber, Gaillard. Drafting the article: Mortazavi, Elliott. Critically revising the article: Oluigbo, Mortazavi, Elliott, Schreiber, Gaillard. Reviewed submitted version of manuscript: Oluigbo, Mortazavi, Elliott, Phan. Approved the final version of the manuscript on behalf of all authors: Oluigbo. Statistical analysis: Mortazavi, Elliott. Study supervision: Oluigbo.

\section{Correspondence}

Chima O. Oluigbo: Children's National Hospital, Washington, DC. coluigbo@childrensnational.org. 